\title{
Multitouch Tables for Collaborative Object-based Learning
}

\author{
Jacob George ${ }^{1}$, Eric de Araujo ${ }^{2}$, Desiree Dorsey ${ }^{3}$, \\ D. Scott McCrickard ${ }^{1}$, and Greg Wilson ${ }^{1}$ \\ ${ }^{1}$ Department of Computer Science and Center for Human-Computer Interaction, \\ Virginia Tech, Blacksburg, VA, USA \\ ${ }^{2}$ Department of Information Technology and Department of Philosophy, Bethel College, \\ Mishawaka, IN USA \\ ${ }^{3}$ Department of Psychology, Bennett College, Greensboro, NC USA \\ \{jacobgc, mrwilson, mccricks\}@vt.edu, eric.dearaujo@bethelcollege.edu, \\ ddorsey@bennett.edu
}

\begin{abstract}
Multitouch technology on tabletop displays allows children to interact with digital objects in collaborative activities. This paper explores both evolutions in hardware and opportunities in software toward supporting the engagement of children, with consideration of impact on user interfaces. We outline a demonstration of our Multitouch Education Table (MET), a portable hardware system and virtual card game suite targeted for use by elementary school students.
\end{abstract}

Keywords: multitouch, tabletop, children, education, evaluation

\section{Introduction}

Multitouch systems make possible multiple simultaneous inputs not only from a single user but from multiple users. Chronicled in a report from Buxton [1] and popularized in Jeff Han's 2005 UIST paper [2], multitouch is now widely found in devices from Apple, Microsoft, Google, and others. Researchers and practitioners have developed image manipulation, business applications, games, and, most recently, educational applications (e.g. [3]) to take advantage of the technology. This paper explores the promise that lower prices and greater portability can lead to development of tools for early education.

We discuss hardware features that can yield a low-cost portable tabletopparticularly important in educational settings when cost is a major issue. We describe the advantages to a do-it-yourself approach that leads to modularity and lightweight hardware solutions, and we demonstrate how that can facilitate the development of multitouch applications for student use.

To demonstrate the potential of multitouch in the classroom, we show activities that make use of multitouch digital cards. The card paradigm supports real-world 
card behaviors (e.g., moving and flipping) but also virtual-world behaviors like linking and mapping.

We provide details about a lab experiment that compared multitouch and traditional desktop platforms. Performance metrics were collected for linking tasks, including time to make a match, number of correct responses, and number of errors. In addition, participants took pre-test and post-test surveys to measure knowledge, preference, and additional feedback.

Finally, our paper also reports on reactions of children to multitouch technology. The possibility for multiple students to interact with the multitouch system might prove more cost effective than providing each student access to an individual computer and support collaborative educational goals. As this type of technology makes its way into the classroom, it holds potential to facilitate "learning by doing" for groups of children [3] through support for object-based manipulation.

\section{Customizing hardware for education}

Multitouch table configurations vary in shape, size, and construction. Two commercial examples include the Microsoft Surface and Smart Technologies' SMART Table. The former is used in a variety of environments, while the latter is specifically designed for education. However, these systems face barriers to adoption in educational settings because of their low portability, lack of modularity, and high cost [4]. The goal of MET's hardware design was to provide a portable, modular, and low-cost multitouch display.

The portability of MET is important in an educational setting where the table may need to be shared between classrooms and buildings. To achieve a highly mobile system the MET is built of lightweight materials such as hard-board panels, wooden legs, and a wooden base. These pieces along with the acrylic top, and electronic components (computer, projector, and camera) collectively weigh 70 pounds and can be transported individually. This makes it more portable than the 150-180 pounds seen in most commercial products (which must be transported as a single unit).

The ability to disassemble the unit contributes to its modular design where individual components can be replaced; for example, a single table frame in a school system could utilize projectors already present in the classrooms of each school. This would eliminate the need to transport a projector when bringing MET to the schools, allowing it to be more easily shared among classrooms. (See Fig 1.)

The Do-It-Yourself (DIY) approach that helped make MET portable and modular also produced it for under $\$ 3,000$. This makes it a cost effective alternative to the $\$ 6000$ to $\$ 12,000$ (or more) commercial alternatives. In addition to the reduced cost and improved portability and modularity, improvements in projector and camera technology - with smaller throw distance-could yield a table smaller than the current 45 inches and lighter than the current 70 pounds. LCD displays instead of a projector hold further benefits. 


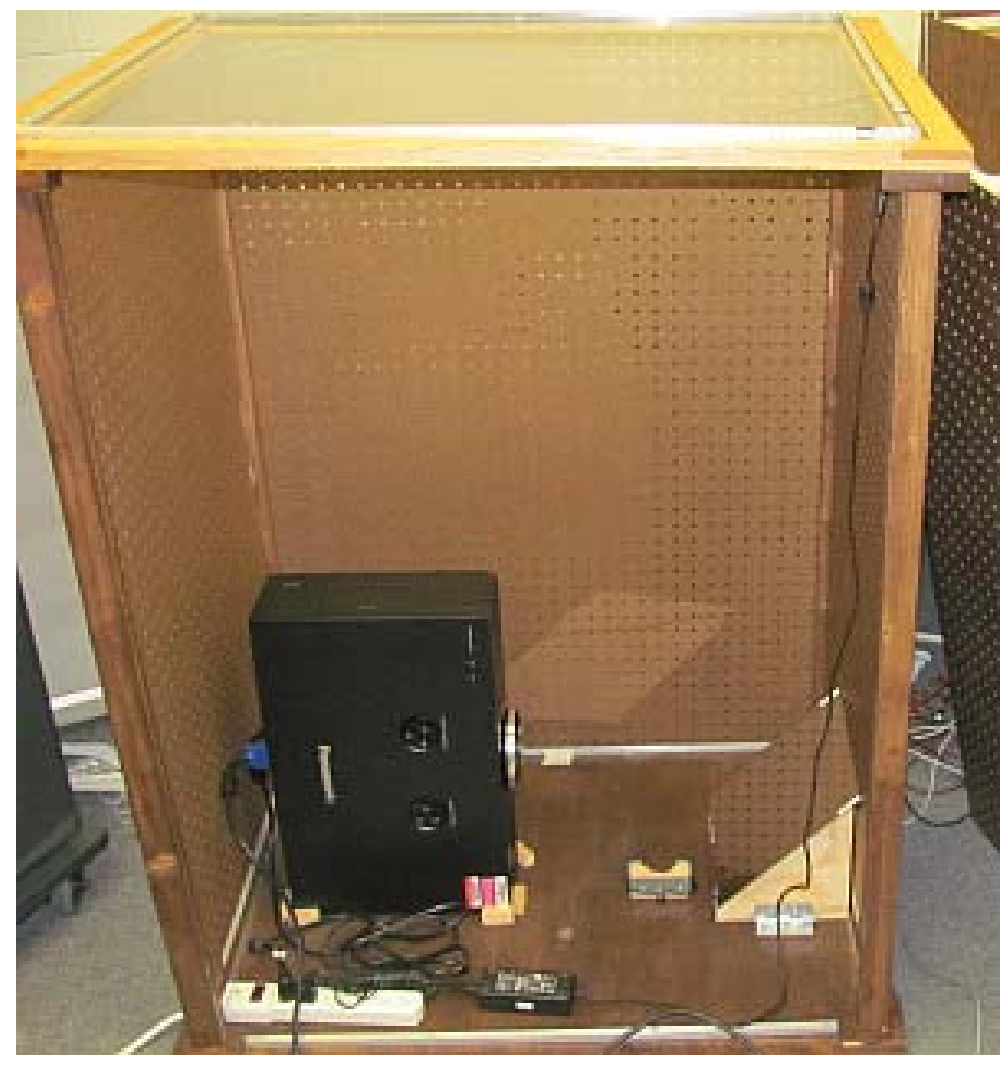

Fig. 1. A side view of the open MET displaying its internal components, including the projector, wiring and power sources, and mirror.

\section{Digital card activities}

The decrease in price and increase in availability of multitouch tables and similar technologies has resulted in an increase in software for educational settings, including efforts by and for Microsoft Surface, SMART Table's Interactive-Learning Center, Infusion's Education Suite, and other commercial and academic efforts. Our attempts have focused on digital cards, virtual knowledge artifacts that can be directly manipulated by users. Prior work suggests that multitouch artifact-based interactions will result in more interaction and fewer negative interactions [3]. We seek to reinvent experiences with physical cards and single-touch applications, but in so doing, we must be careful not to duplicate the paradigms of physical cards or single touch-respecting the novelty of the platform. Like physical cards, they can be moved, flipped, and can include a combination of text or images. Our MET games include pairing and grouping activities targeted to support children's desires to both 
collaborate and compete, and that support teachers' need to tailor the applications for their topics.

Our extension of the card paradigm provides unique advantages over physical cards. By storing the content of the cards in an XML document, the learning activities have been abstracted from the specific educational content. This flexibility supports the use of these cards for teacher-selected content that could be modified by entering topic elements (e.g., animal-country pairs, or animal life-cycle sequences like egglarva-pupa) and relationship types (explained next) describing associations between elements.

Four relationship types, represented by similar multi-handed gestures that seem to come naturally to young children, can support a wide variety of learning applications. Linking connects cards that share an unordered relationship (e.g., animals and countries, or inventors and inventions), accomplished by dragging cards so that they overlap. Combining builds upon linking in that cards with a certain relationship are brought together to form a new card (e.g., combining colors like red and blue to create purple). Mapping associates objects with a position or region on the screen, like objects to a map or other fixed category. In sequencing, the left-to-right or top-tobottom ordering of objects has meaning, important for subject areas like animal lifecycles and ordered historical events. See Figure 2 for an example of the "link" behavior.

We have built numerous card-based applications for the MET, several of which are highlighted in the remainder of this section. The Math Match game, targeted for students in grades 7-12, provides an environment in which students learn about concepts like order of operations and algebraic concepts. Participants in the game can play by themselves, compete with others, or cooperate toward finding the right answer to problems. The Animal-Continent game, targeted at young elementaryschool-age student, asks participants to match animals to the continent in which the animal is naturally found (see Figure 2). When a card is released on the correct continent on a map, it shrinks and locks into place. The Inventors-Inventions game is similar to the Animal-Continent game in that matched pairs are created. Different card sets can target different disciplines (e.g., math, sciences, engineering) and different demographics (e.g., African-Americans, women). Other games allow participants to create matchings, links, or orderings among colors and shapes. 


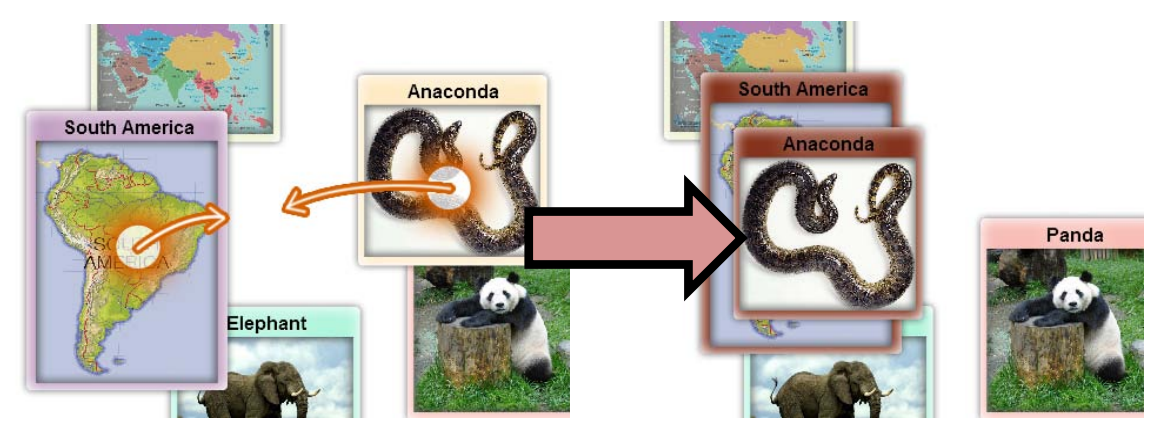

Fig. 2. South America and the Anaconda card are dragged toward each other (left), then when the cards overlap, they "link" and provide visual and auditory feedback (right)

\section{User study: Multitouch tables and desktop displays}

A user study was performed to explore performance and enjoyment of the multitouch table environment in a controlled lab-based setting. This study compared performance and preferences for 11 college undergraduate students on three card game activities using our multi-touch table (MET) and using a traditional desktop computer. This user study appears in full detail in another paper [5]; the approach and key results are summarized here.

The students were separated into two groups, and they were asked to work individually on a series of three activities: a color-matching activity, a shapematching activity, and an inventor/invention-matching activity. Students in one group started with the desktop activities first, and the other group started with the MET. Students in both groups alternated between the desktop and MET until they completed two trials for each activity type on each system. Statistics like the total game time and amount of time the student to complete each individual match were stored.

Among the results, one stood out: users took significantly less time to complete activities using a traditional computer desktop than the MET $(p=0.0145)$. However, user comments comparing the MET and desktop games showed that people preferred using the MET, finding the game more enjoyable on that platform and expressing a desire to see it in classroom settings. It is possible that the lack of familiarity and other technical difficulties and limitations degraded performance with the MET, and that with practice the participants could perform as well or better with the MET as with a traditional desktop system. In addition, technological advances in multitouch surfaces may lead to increased performance. 


\section{Multitouch in the field: Notes from visits with student groups}

To understand the needs and desires of our target user population, we frequently conduct presentations and hands-on demos with students and educators. It was important to test the durability and portability of the table (to see if it could be disassembled, moved to a different location, and reassembled with ease), test the usability of the card matching applications, as well as interview and gauge the interest of the children in using a multitouch table and possible applications. Figure 3 is illustrative of our experiences in the field.

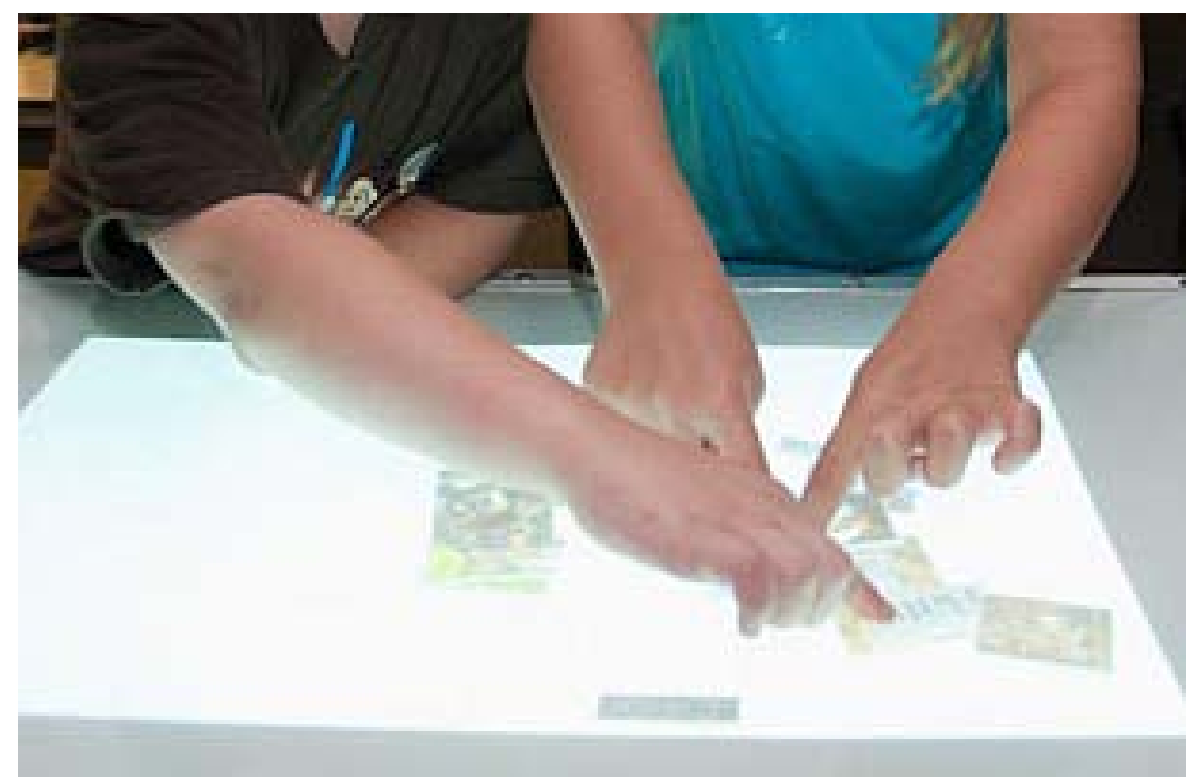

Fig 3: Typical behavior among children using the MET, with multiple people, hands, and fingers all manipulating digital objects simultaneously.

The first experience with demoing the table came at the Destination ImagiNation Regionals in Salem, Virginia. Destination ImagiNation is a non-profit organization that features teams of students ranging from elementary school to college competing in creative problem solving challenges. This description made this group a perfect setting to setup the table. The table was set up as a side attraction for the elementary children to experience in between their challenges. No applications had been created at the time, so small demo applications were run instead. These include a chalk board application and a basic visualization application where the children could see abstract visualizations wherever they touched on the surface.

Because this was the first time demoing the table in public, there were some minor obstacles to overcome. There was an issue of finding a good position for the table, because it had to be located near a power source. The table also could not be directly under a light source because it interfered with what the table processed as touches. 
Our two options became setting up in a high traffic where it was hard for anyone to use the table or further down the hallway where it would not be immediately seen by guests. Although we were not in an ideal location, there were still 10-12 kids that visited the table. They enjoyed playing the demos on the table and stayed at the table for approximately 15-20 minutes. Because there was only one researcher there who was tasked with making sure the table function properly, there was not a chance to interview the children more formally.

The summer camp visit was conducted at the STEM Fun! Camp hosted by the Institute for Advanced Learning and Research in Danville, Virginia. During the camp, elementary school aged children are exposed to many new technologies and participate in science experiments with the goal of getting them interested in and motivated about STEM (Science, Technology, Engineering, and Math) subjects.

The visit consisted of a formative intervention with 14 participants in the camp, who explored a classification (linking) activity. The participants were brought to the multitouch table in pairs. To begin the session, the participants were given an explanation on what a multitouch table was. They were given a high level overview of how the multitouch table technology worked while being shown the inside parts of the table. The participants were then able to touch the screen and view a visual representation of how the table was sensing and responding to their touches. The children then worked together to match sets of seven or eight animals to their countries or continents of origin (e.g., kangaroos to Australia) - which they were able to complete in about a minute per set, with minimal assistance (shown in Figure 2). Afterwards, the children participated in a short discussion in which they were asked if they thought the multitouch table would be useful in their classrooms and if so, what school subjects would it be most beneficial.

The students reported only minor problems using the technology (primarily related to the need with our technology to touch with fingers or hands and not fingernails), and almost all would be willing to use the table on a frequent and regular basis as part of their learning activities. One issue with the table is that it was frequently too tall for the participants to be able to see and touch the whole surface. To rectify this, we had to use chairs for the children to stand on so that they could have access to the entire table. Obviously, this is not the safest approach to this issue. For future prototypes, we would have to take this issue into consideration and lower the height of the table so that it is usable for all ages and heights. Another issue with the durability of the table was that as a result of the touching surface being made with tracing paper, after extended use the surface could become sticky, dirty, or torn. This issue can cause the table to process "phantom" touches. To counter this problem we changed the touching surface to a more stable Rosco Grey material. This material also helped to block the extra light that the table was processing.

Over half of the participants had previous experiences with using a multitouch device. This experience mostly came from (their parent's) phones, iPods, and other games. All the participants believed that the table would be useful in classroom. The subject that was selected the most by the children for being most benefited by a multitouch table was math followed by science. It is possible that these subjects were already at the forefront of the children's minds since they were attending a STEM camp. This could have led to them to not thinking about other subjects such as history, geography, and literature that could also benefit from multitouch table 
applications. Often during the session, instead of having the children work together to match the animals to the countries, they were told to be competitive and work fast to have the most matches in the first time. This new twist seemed to motivate the children more and resulted in more laughter, faster matches, and more errors. This lack of enthusiasm for collaborating could be why around half of the participants said they would rather work with a partner than alone-perhaps highlighting the need to create better collaborative applications!

Another intervention took place during the Harding Avenue Elementary School Computer Club. This club meets weekly for an hour, and during one of the weekly sessions a 3-person team brought the MET to the session. There were $124^{\text {th }}$ and $5^{\text {th }}$ grade students in attendance ( 7 boys and 5 girls; 10 white and 2 African-American, both females). They had the opportunity to use the MET or to use a game-creation program. To oversee the activity, there was one administrator, two MET developers, and eight undergraduate students.

There was excitement about the MET among students, particularly the boys. The boys showed little hesitation in trying new gestures with the table, grabbing and manipulating the virtual cards in ways not dictated by the rules of the game or activity. For example, the boys were more excited to explore ways in which to disrupt actual gameplay. They would resize a card to fill up the whole board, place objects incorrectly in other boys' bins, or populating the display with an excessive number of cards-laughing enthusiastically while doing so. This would draw attention to the novel technology, but not in a particularly helpful way.

The girls tended to be more hesitant, seeking to learn the rules and to wait for a demo from a facilitator before starting the game. The boys dominated the time with the MET early on, while the girls clustered in one side of the room for much of the session. At first it appeared that they did not like the competiveness of the game that we were playing at the time. After being asked if they wanted to play with the MET, they initially showed disinterest, but later the two African American girls came to the table and took a leadership role in playing the games. It appeared that the girls took the rules of the games very seriously and they wanted to play the games the way they were supposed to be played. During this time the other three girls did not approach the table; it was unclear whether this was because of the number of kids were at the board or because of dynamics with the kids were at the table (the two African American girls particularly seem to aggressively boss around the other children). After the two African American girls and some of the boys left, the other three girls came to the table to see what it was happening. When they were introduced to the table they did not try to play any of the games out right they just played with the cards moving them around and resizing them. They appeared to have no interest in any competiveness that the inherent in the game.

When the students first started to interact with the board, they would only use one finger on the board—as they would with a mouse and cursor icon—even though it was demonstrated to them how to use multiple fingers or hands. They did not start using two hands at a time until they were presented with an action that required to hands. After experiencing the benefits of multitouch, the students used multiple fingers and hands frequently. By the end of the session, all of the students were very social in their interactions, talking and looking at others' activities and games. The 
MET was a good match for these activities since there were many hands on the table at any time.

With respect to the portability and durability of the table, we found that disassembly and reassembly of the table usually takes about 15 minutes with one person. The marks placed on the sides, bottom, and top of the table make it easy to assemble the parts in the correct positions. Transporting the table in a vehicle is simple because you can organize the table where the bottom and the sides are stacked on top of each other. The surface top, because it has a glass and sensitive surface, can still on top of the other parts. Because the sides are made of wood, constant adding and removal of the sides can cause the edges of the sides to start to splinter. Another issue is keeping up with all the cables, because the computer, projector, and the surface top all have power cables it can be cumbersome to keep up with all of them. Having a backup computer is essential, in case issues occurred with the computer during travel. Because the table is not completely multitouch, there is also a need to transport a keyboard and mouse as well. An ideal situation would be to have the table that is completely multitouch or at least a cordless keyboard and mouse. There also may be a need to carry a separate extension cord for times where the table cannot be directly near a power source. There were numerous of occasions during demos and school visits where the table was limited to where it could be located because it had to be close to a power source.

\section{Conclusions and future directions}

Multitouch technology supports the manipulation of digital objects using multiple touches-either from a single person or many. This paper explored how our selection of a small number of multitouch gestures representing relationships among objects can support learning applications, toward a toolkit where teachers could create their own digital cards that match their curriculum. Our extension of the card paradigm provides unique advantages over physical cards. By storing the content of the cards in an XML document, the learning activities have been abstracted from the specific educational content. This flexibility supports the use of these cards for teacherselected content that could be modified by entering topic elements (e.g., animalcountry pairs, or animal lifecycle sequences like egg-larva-pupa) and relationship types describing associations between elements.

An area of particular interest for future investigation is in the development of collaborative educational activities for multitouch tables. While many desktop computer applications are inherently single-user, a multitouch table provides opportunities to learn about diverse topics, such as history [6], math [7], music [7], and even basic social skills [8]. Other future work includes quantitative and qualitative study of the multitouch gestures and point and click interfaces, and distribution of applications. Our interventions have demonstrated the promise of multitouch, but a more in-depth exploration is needed. It is our expectation that follow-up work will result in an understanding of how and when technology like multitouch should best be used; e.g., in classroom teaching, as part of after-school programs, or through extracurricular activities like scouting or $4 \mathrm{H}$ clubs. 


\section{Acknowledgements}

Thanks goes to the U.S. National Science Foundation (NSF) for grants IIS0851774 and CNS-0940358 that supported this work. The opinions in this paper are our own and are not necessarily shared by the NSF.

The authors would like to thank the many people who helped contribute to the construction of the table and to early software instantiations, particularly Goldie Terrell, Stacy Branham, Kristin Whetstone, Chase Carroll and Keith Manville. Thanks also to the many students who used MET, and to the teachers and administrators for their time and insight.

\section{References}

1. Buxton, B. Multi-touch systems that I have known and loved. Unpublished manuscript. Downloaded from www.billbuxtoncom/multitouchoverview.html 21 Jan 2011.

2. Han, J. Low-Cost Multi-Touch Sensing through Frustrated Total Internal Reflection. In: Proceedings of UIST (2005)

3. Rick, J., Harris, A., Marshall, P., Fleck, R., Yuill, N., and Rogers, Y. Children Designing Together on a Multi-Touch Tabletop: An Analysis of Spatial Orientation and User Interactions. In Proceedings of IDC (2009)

4. Vallis, K. and Williamson, P. Build Your Own Board. Learning \& Leading with Technology. (2009)

5. Dorsey, D., George, J., de Araugo, E., Wilson, G., and McCrickard, D. S. Multi-touch tables for collaborative learning. In Proceedings of the 2010 Annual Biomedical Research Conference for Minority Students (2010)

6. Ardito, C. Costabile, M.F., and Lanzilotti, R. Gameplay on a multitouch screen to foster learning about history. In: Proceedings of the International Conference on Advanced Visual Interfaces (2010).

7. Yu, X. Zhang, M., Ren, J. Zhao, H, and Zhu, Z. Experimental development of competitive digital education games on multi-touch screen for young children. In: Proceedings of Entertainment for Education and $5^{\text {th }}$ International Conference on E-learning and games (2010)

8. Piper, A.M., O’Brien, E., Morris, M.R, and Winograd, T. SIDES: A cooperative tabletop computer game for social skills development. In: Proceedings of CSCW (2008) 